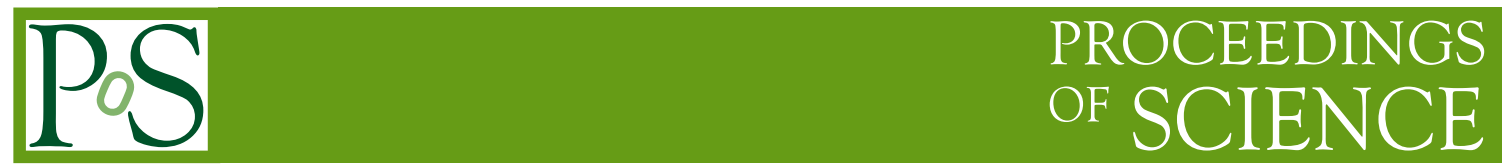

\title{
Top physics at ATLAS
}

\author{
Saverio D'Auria*† \\ University of Glasgow \\ E-mail: saverio.d'auriaecern.ch
}

This paper is an overview of recent results on top-quark physics obtained by the ATLAS collaboration from the analysis of $p-p$ collisions at 7 and $8 \mathrm{TeV}$ at the Large Hadron Collider.

LHC on the March

20-22 November 2012

Institute for High Energy Physics, Protvino, Moscow region, Russia

\footnotetext{
*Speaker.

${ }^{\dagger}$ On behalf of the ATLAS collaboration
} 


\section{Introduction}

At the Large Hadron Collider (LHC) the standard model is being tested at the frontier of energy, and so far no deviation has been found. The SLC and LEP result on the number of light neutrinos [1], together with the recent LHC results on the cross section of the Higgs-like particle [2, 3] seem to indicate that there is very little room for new families of fermions. The mass of the Higgs boson and the mass of the top quark are very important parameters of the standard model, and a precise measurement of both will give an indication of the stability of the standard model at very high energies [4]. The measurement of the Higgs boson mass alone will not be enough to pin down the model. The LHC is on the march, but this march has been in a desert, if we exclude the experimental observation of standard model particles. Of course, the discovery of the Higgs boson was a formidable achievement, but we all look forward for an oasis after the next dune of the standard model. Measurements of the top-quark properties can shed direct or indirect light onto new physics.

This overview will describe some of the recent top mass measurements, then productionrelated observables: cross sections and spin correlations, the electroweak top production and searches for $t \bar{t}$ resonances. The top quark production is also one of the main background to searches for new physics, in particular when the top quark is produced with additional jets. This talk covers only some of the top physics results achieved by the ATLAS Collaboration. An updated and more complete list is available at the ATLAS public top result website [5]. The ATLAS detector and its performance is described elsewhere [6, 7]. In 2010 and 2011 the LHC has delivered $4.7 \mathrm{fb}^{-1} p-p$ collisions at $\sqrt{s}=7 \mathrm{TeV}$, and at the time of this workshop $14 \mathrm{fb}^{-1}$ at $\sqrt{s}=8 \mathrm{TeV}$.

The measurements of top quark properties can be performed using several decay channels of the two top quarks. The all-hadronic decay mode accounts for $45.7 \%$ of the branching fraction, but suffers of trigger limitations and high background. The case in which one $W$ boson decays into an electron or muon and its corresponding neutrino (or antineutrino) and the other $W$ decays into quark jets is refererred to as "lepton+jets" channel and accounts for $28.8 \%$ of the branching fraction. The decay modes involving one or more $\tau$ lepton occur $21.1 \%$ of times and may contaminate the lepton+jets modes. In turn, analyses involving $\tau$ can further be cathegorised according to the $\tau$ lepton decay modes. The case in which both $W$ from the top quark pair decay into $e v$ or $\mu \nu$ is referred to as the dilepton decay channel, which accounts for only $4.5 \%$ of the branching fraction, it has the advantage of being cleaner from trigger and backgound point of view, but the two undetected neutrinos make the kinematics under-constrained.

\section{Top Quark mass}

The measurement of the mass of the top quark is presently dominated by systematic uncertainties. The most recent ATLAS analysis at the time of this workshop is using the dilepton decay mode for $4.7 \mathrm{fb}^{-1}$ collected at $7 \mathrm{TeV}$ centre of mass [8]. This analysis requires two jets originated from a $b$-quark and two leptons $\left(e^{ \pm}\right.$or $\left.\mu^{ \pm}\right)$. There are two combinations pairing a lepton with a b-jet. The missing transverse momentum is split between two neutrino momentum, and each of the neutrino is used to calculate the top transverse mass, for each lepton-b-jet pair. The minimum of the two values of transverse mass is chosen, as well as the minimum over the lepton-b-jet pair. The transverse mass 


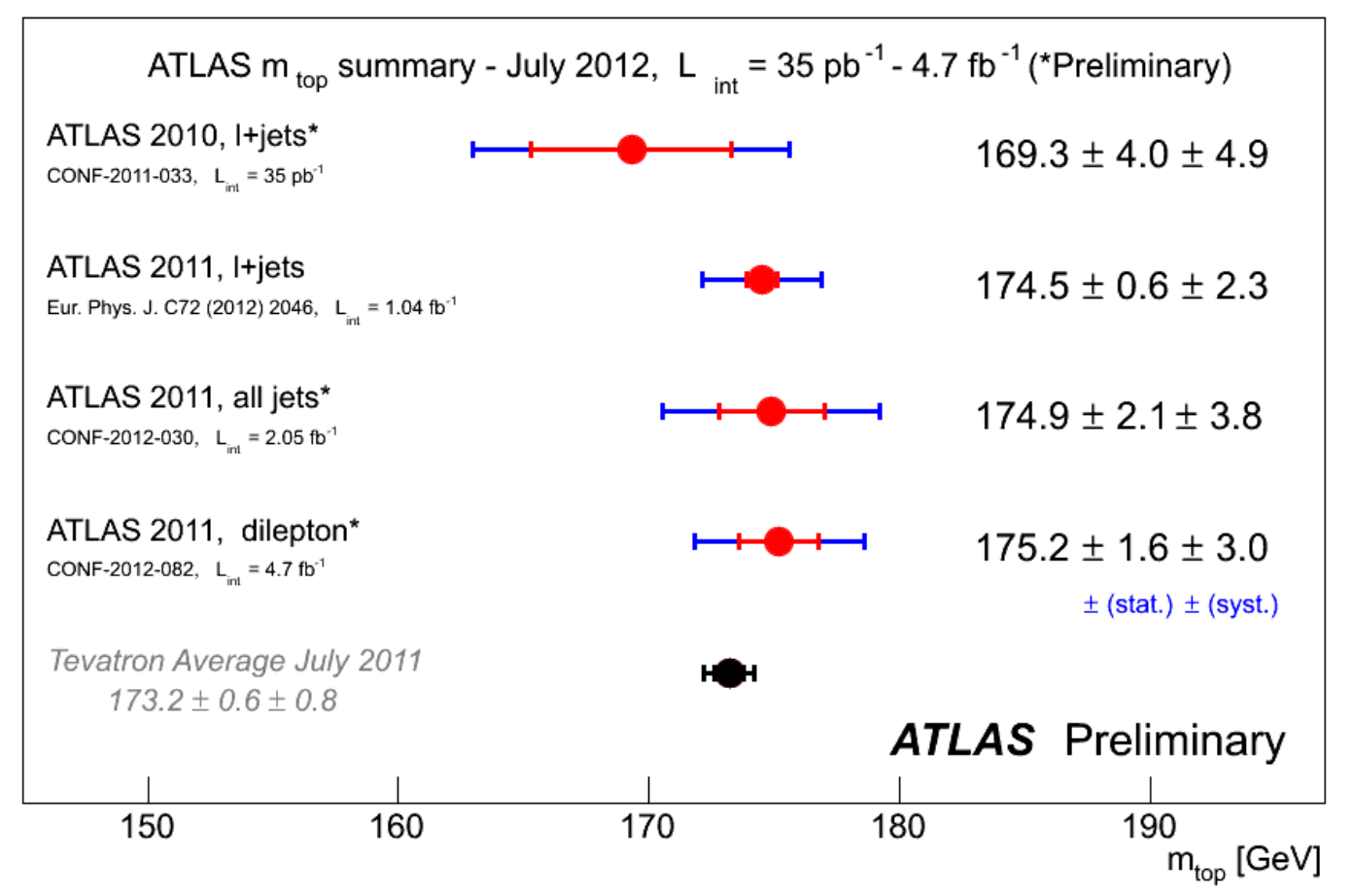

Figure 1: A summary of the measurements of the top quark mass by the ATLAS collaboration.

is proportional to the top quark mass. A Monte Carlo simulation is used to calibrate the method. The resulting top mass is $m_{t}=175.2 \pm 1.6(\mathrm{stat})_{-2.8}^{+3.1}$ (syst) GeV. The main systematic uncertainties originate from the jet and b-jet energy scales, the colour reconnection and the Monte Carlo models. The most precise ATLAS analysis at the time of this conference has been made with the lepton+jets decay mode [9] and is $m_{t}=174.5 \pm 0.6$ (stat) \pm 2.3 (syst.) $\mathrm{GeV}$. The analysis of the all-hadronic decay mode [10] using $2.05 \mathrm{fb}^{-1}$ resulted in a value of $m_{t}=174.9 \pm 2.1$ (stat) \pm 3.8 (syst.) GeV. A summary of the measurements of the top quark mass is shown in fig. 1, where the Tevatron average [11] $m_{t}=173.2 \pm 0.6$ (stat) \pm 0.8 (syst) $\mathrm{GeV}$ is also reported, for comparison. The most recent combination of Tevatron results is $m_{t}=173.18 \pm 0.56$ (stat) \pm 0.75 (syst) [12].

\section{Production cross sections of top anti-top pairs}

The production cross section of $t \bar{t}$ pairs has been measured at 7 and $8 \mathrm{TeV}$ c.m. energy. The only analysis with data at $\sqrt{s}=8 \mathrm{TeV}$ [13] currently selects lepton+jets events and requires a muon $p_{T} \geq 40 \mathrm{GeV}$ or an electron $E_{T} \geq 40 \mathrm{GeV}$, at least 3 jets and at least one of them to be $b$-tagged. Also missing transverse energy is required. This analysis uses a discriminant function based on the lepton pseudorapidity $\eta=-\ln \tan (\theta / 2)$ and the transformed aplanarity $A^{\prime}=\exp \left(-12 \lambda_{3}\right)$ where $\lambda_{3}$ is the smallest eigenvalue of the sphericity tensor. The discriminant distribution is fitted. The main surce of systematic uncertainty is the Monte Carlo modeling of the signal, for a total of $11 \%$ which is due to the production process modeling in MC@NLO [14, 15], AlpGen [16] and POWHEG [17], the parton shower model in POWHEG and Pythia [18] compared to POWHEG and 
HERWIG [19] and the systematics due to parton distribution functions. The initial and final state radiation contributes for $4 \%$. The cross section at $\sqrt{s}=8 \mathrm{TeV}$ is $\sigma_{t \bar{t}}=241 \pm 2$ (stat.) \pm 31 (syst.) \pm 9 (lum.) pb. which can be compared to the theoretical prediction of $\sigma_{t \bar{t}}$ (th.) $=238_{-24}^{+22} \mathrm{pb}$ [20].

The top pair cross section at $7 \mathrm{TeV}$ has been recently measured in the lepton+jets channel selecting only events with semi-leptonic decay of $b$-hadrons [21]. This way the systematic uncertainties related to the b-tagging were not correlated with respect to previous analyses. The resulting cross section is $\sigma_{t \bar{t}}=165 \pm 2$ (stat.) \pm 17 (syst.) \pm 3 (lum.) pb. Recent measurements of the $t \bar{t}$ cross section at $7 \mathrm{TeV}$ include the all-hadronic decay channel [22] using $4.7 \mathrm{fb}^{-1}$ : $\sigma_{t \bar{t}}=168 \pm 12$ (stat.) ${ }_{-57}^{+60}$ (syst.) \pm 6 (lum.) pb and two analyses, which use hadronic $\tau^{ \pm}$decays: one $\tau_{\text {had }}+$ jets [23] with $1.67 \mathrm{fb}^{-1}$ and another using $(e$ or $\mu)+\tau_{\text {had }}+$ jets [24] with $2.05 \mathrm{fb}^{-1}$. A summary of the $t \bar{t}$ cross section measurements at $\sqrt{s}=7 \mathrm{TeV}$ is shown in fig. 2. The different relative error on the luminosity reflects our best knowledge of the calibration uncertainty at the time when the preliminary measurement was released.

The differential cross section as a function of the transverse momentum and rapidity has been measured in the lepton+jets channel at $7 \mathrm{TeV}$ [25]. The unfolded distributions have been calculated by inverting the response matrix. No deviation from the standard model has been found.

\section{Electro-weak top production}

The single top production cross section has been measured at $7 \mathrm{TeV}$ [29] and at $8 \mathrm{TeV}$ [30] in the t-channel production mode, where the top is produced in association with a light-quark jet. The ATLAS measurement uses events where the $W$ from the top decays to a lepton and neutrino. The cross section at $8 \mathrm{TeV}$ has been measured to be $\sigma_{t}=95 \pm 2$ (stat.) \pm 18 (syst.) \pm 3 (lum.) pb. This measurement has been translated to a direct measurement of the CKM matrix element, giving $\left|V_{t b}\right|=1.04_{-0.11}^{+0.10}$. The ratio of top to anti-top cross section has been measured at $7 \mathrm{TeV}$ using $4.7 \mathrm{fb}^{-1}$ [31]. The associated $W t$ production cross section has been measured at $7 \mathrm{TeV}$ using $2.05 \mathrm{fb}^{-1}$ and no b-tagging information [32] and is $\sigma_{W t}=16.8 \pm 2.9$ (stat.) \pm 4.9 (syst.) pb. The corresponding CKM matrix element is $\left|V_{t b}\right|=1.03_{-0.19}^{+0.16}$. The search for the s-channel $t \bar{b}$ production at the time of this conference has been performed using $0.7 \mathrm{fb}^{-1}$. This signal has the same final state as the $W^{ \pm}$Higgs associate production with $H \rightarrow b \bar{b}$. The main backgrounds for this analysis is $W+$ jets and $t \bar{t}$. The limit to the production cross section is $26 \mathrm{pb}$.

All measurements are compatible with the approximated next-to-next-to-leading order calculations [33].

\section{Search for resonances}

An enhanced production of s-channel single top $t \bar{b}$ could be due to the existence of a new vector boson $W^{\prime} \rightarrow t \bar{b}$. A limit to the $W^{\prime}$ mass has been set to $m_{W^{\prime}}>1.13 \mathrm{TeV}$ at $95 \%$ confidence level using $1.04 \mathrm{fb}^{-1}$ at $7 \mathrm{TeV}$ [34].

A direct search for resonances decaying to $t \bar{t}$ has been performed in the full hadronic decay mode using $4.7 \mathrm{fb}^{-1}$ at $\sqrt{s}=7 \mathrm{TeV}$ [35]. In order to do this in the LHC environment in which several $p$ - $p$ collisions take place at the same bunch crossing it is essential to keep under control the effects of pile-up. The average number of collisions per bunch crossing has increased from 


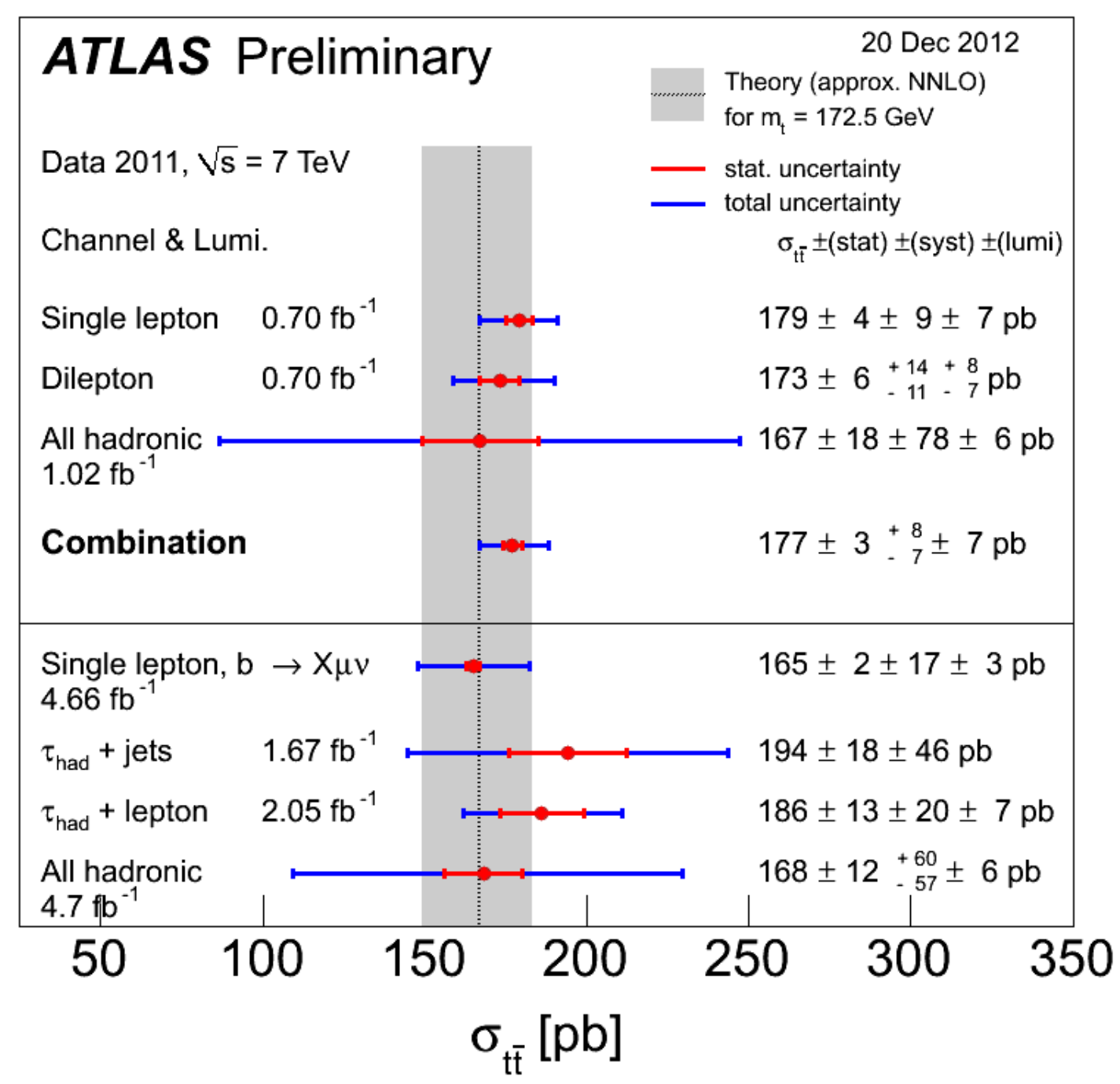

Figure 2: Summary of measurements of the top-pair production cross-section at $\sqrt{s}=7 \mathrm{TeV}$, compared to the corresponding theoretical value based on an approximate NNLO calculation [20]. The upper part of the figure shows the measurements that are averaged to give the combined value; the lower part includes more recent measurements, which are described in the text; they are not included in the combination.

9.1 in 2011 data to 20.0 in 2012 data at the time of this workshop $\left(14.0 \mathrm{fb}^{-1}\right)$. The Jet Vertex Fraction (JVF) is used to associate a jet to a primary vertex. It is defined as the fraction of the scalar sum of transverse momentum of the tracks matched to a jet which is contributed by the tracks originating from the same vertex [36]. The analysis searching for resonances uses two methods to identify hadronic decays of top quarks which are produced at $p_{T} \gtrsim 200-300 \mathrm{GeV}$ : the so-called HEPTopTagger [37, 38] and the Top Template Tagger [39, 40]. The former uses jets reconstructed with the Cambdridge-Aachen algorithm [41], looking for a substructure in jets with large distance parameter $\mathrm{R}=1.5$. These are called "fat-jets". The latter uses a large set of pattern of energy deposits from hadronic decay of top quark to identify the best match to the experimentally 
observed jets and discriminate against light-quark and gluon jets. The jet algorithm used in this case is anti- $k_{t}$ [42] and it is optimised for top-quark $p_{T}>450 \mathrm{GeV}$. Triggering on hadronic events is not trivial. All the jet triggers were based on anti- $k_{t}$ algorithm. The HEPTopTagger analysis used a trigger based on the presence of one jet with $E_{T}>100 \mathrm{GeV}$ and cuts based on the sum of the transverse energy or on the multiplicity of jets. The Top Template Tagger analysis is based on events with at least one jet with $E_{T}>240 \mathrm{GeV}$. The event selection is based on jet- $p_{T}$ and on angular distance with respect to b-jets. The data were compared to models of a leptophobic $Z^{\prime}$ and massive gluons (Kaluza-Klein). The $Z^{\prime}$ has been exluded in a mass range between 0.70 and $1.00 \mathrm{TeV}$ and between 1.28 and $1.32 \mathrm{TeV}$. The KK-gluon has been excluded in the mass range 0.70 to $1.62 \mathrm{TeV}$. Other ATLAS analyses have been published which exclude $Z^{\prime}$ in the range 0.5 to $1.15 \mathrm{TeV}$ and $\mathrm{KK}$-gluons in the range 0.5 to $1.5 \mathrm{TeV}$ [43] [44]. Also the measurement of the differential $t \bar{t}$ cross-section [25] shows no deviation from a non-resonant production. More seaches for new physics by the ATLAS collaboration are reported elsewhere in these proceedings [26] and are available from the collaboration web pages [27, 28].

\section{Polarization measurements}

The polarization of $W$ bosons originating from top quark decays has been measured both in the lepton+jets and the dilepton channels using $1.04 \mathrm{fb}^{-1}$ [45]. The polarization can be longitudinal, right-handed and left-handed, and the fraction of events with a $W$ in a given polarization state can be measured with the distribution of the helicity angle $\theta^{*}$ in the $W$ boson rest frame. It is defined as the angle between the momentum direction of the charged lepton and the reversed momentum direction of the b-quark from the decay of the top quark which originated the $W$. Two methods have been used: the template and the angular asymmetries. The first compares the measured $\cos \theta^{*}$ distribution with templates from Monte Carlo simulations with different $W$ helicities. The second method calculates the asymmetries of the $\cos \theta^{*}$ distribution after correcting for the background. The asymmetries are related to the helicity fractions. The measured fractions using the two methods were combined, resulting in the following values for longitudinally, left- and right-handed polarization:

$$
\begin{aligned}
& F_{0}=0.67 \pm 0.07 \\
& F_{L}=0.32 \pm 0.04 \text { and } \\
& F_{R}=0.01 \pm 0.05
\end{aligned}
$$

All measured values are in agreement with the standard model predictions. Any deviation from these predictions could be due to new physics, so this measurement allowed to put limits on the parameters of an effective Lagrangian describing a hypothetical new interaction.

No polarization of the top quark is expected in $p-p$ collisions from $g g$ or $q \bar{q}$ non-resonant production, so any polarization is an indication of a non-standard production mechanism. The top polarization has been measured using $4.66 \mathrm{fb}^{-1}$ at $7 \mathrm{TeV}$ in the lepton+jets decay mode [46]. The polarization of the quark is studied in the reference frame where the top is at rest by measuring the angle $\theta_{l}$ of the lepton with respect to the direction of the top-quark momentum in the $t \bar{t}$ rest frame. In order to do so the $t \bar{t}$ system needs to be fully reconstructed. The main systematic uncertainties 
in this case come from the jet energy scale and from signal modeling. The fraction $f$ of positively polarised top quarks has been measured with a fit to the distribution of $\cos \left(\theta_{l}\right)$ obtaining $f=$ $0.470 \pm 0.009$ (stat) ${ }_{-0.032}^{+0.023}$ (syst). The standard model prediction is $f_{\mathrm{SM}}=0.5$.

\section{Top quark production with additional jets}

The measurement of the production rate of additional jets in $t \bar{t}$ events provides an important test of QCD prediction and allows to constrain Monte Carlo generators, whose uncertainties affect all top-related analyses. The production of $t \bar{t}+\mathrm{jets}$ is a background to other physics signals, like the top-Higgs associate production. This gives an additional reason for its detailed study. An analysis based on $4.7 \mathrm{fb}^{-1}$ at $\sqrt{s}=7 \mathrm{TeV}$ [47] has measured the jet multiplicity in top events using the lepton+jets decay mode and has compared it to the following Monte Carlo models: POWHEG+PYTHIA, ALPGEN+PYTHIA, ALPGEN+HERWIG, MC@NLO+HERWIG. The latter tends to underestimate the amount of additional jets for seven or eight jets.

\section{Conclusions}

The ATLAS detector has efficiently recorded good data quality and the LHC has delivered a high integrated luminosity. The study of top quarks can answer fundamental questions on the standard model. This quark is undoubtedly a peculiar particle, with a Yukawa coupling having an order of magnitude of one and it is a powerful probe of physics beyond the standard model. Its production mechanism in $p-p$ collisions is described very well by the standard model, as well as its decay, including the angular distributions. The measurement of the CKM matrix element $\left|V_{t b}\right|$ is showing no surprise. A direct search for resonances decaying to $t \bar{t}$ or $t \bar{b}$ is a powerful probe to model of new physics, but so far no hint of new physics has been found. The large coupling of the top quark to the Higgs boson makes it possible to measure, with more data, the cross section of the associated production of $t \bar{t} H$. The top-production is also a background to many searches for new particles. All the standard model measurements shown in this talk are limited by systematic uncertainties. As more data is being analyzed our understanding of the detector is improving and calibrations are being refined. For some processes the Monte Carlo modeling of the physics is a limiting factor so far. The large LHC luminosity, with up to $40 p$ - $p$ interactions per bunch crossing has added steps and checks to the analyses to cope with high pile-up, but it has been demostrated that such a high luminosity can produce good physics analyses. ATLAS is looking forward to refine the calibrations, improve the analysis methods and use the full statistics.

\section{Acknowledgements}

The author's participation to this conference was supported by the Science and Technology Research Council.

\section{References}

[1] S. Schael et al. [ALEPH and DELPHI and L3 and OPAL and SLD and LEP Electroweak Working Group and SLD Electroweak Group and SLD Heavy Flavour Group Collaborations], "Precision electroweak measurements on the Z resonance”, Phys. Rept. 427 (2006) 257. 
[2] S. Chatrchyan et al. [CMS Collaboration], "Observation of a new boson at a mass of $125 \mathrm{GeV}$ with the CMS experiment at the LHC”, Phys. Lett. B 716 (2012) p. 30.

[3] ATLAS Collaboration, "Observation of a new particle in the search for the Standard Model Higgs boson with the ATLAS detector at the LHC", Phys. Lett. B 716 (2012) p. 1.

[4] G. DeGrassi, S. Di Vita, J. Elias-Miro, J. R. Espinosa, G. F. Giudice, G. Isidori, A. Strumia, "Higgs mass and vacuum stability in the Standard Model at NNLO ", JHEP 1208 (2012) 098, arXiv:1205.6497 [hep-ph] .

[5] https://twiki.cern.ch/twiki/bin/view/AtlasPublic/TopPublicResults .

[6] ATLAS Collaboration, "The ATLAS Experiment at the CERN Large Hadron Collider", JINST 3 (2008) S08003.

[7] ATLAS Collaboration, "The performance of the Atlas detector", Springer, 2010, ISBN 978-3-642-22115-6

[8] ATLAS Collaboration, ATLAS-CONF-2012-082, http://cds.cern.ch/record/1460394 .

[9] ATLAS Collaboration, "Measurement of the top quark mass with the template method in the $t \bar{t} \rightarrow$ lepton+jets channel using ATLAS Data", Eur. Phys. J. C72 (2012) p. 2046.

[10] ATLAS Collaboration, ATLAS-CONF-2012-030, http://cds.cern.ch/record/1431895 .

[11] [CDF and D0 Collaborations], "Combination of CDF and DO results on the mass of the top quark using up to $5.8 \mathrm{fb}^{-1}$ of data", FERMILAB-TM-2504-E, CDF-NOTE-10549, D0-NOTE-6222, arXiv:1107.5255 [hep-ex].

[12] T. Aaltonen et al. [CDF and D0 Collaborations], "Combination of the top-quark mass measurements from the Tevatron collider", Phys. Rev. D 86 (2012) p. 092003.

[13] ATLAS Collaboration, ATLAS-CONF-2012-149, http://cds.cern.ch/record/1493488 .

[14] S. Frixione and B.R. Webber, "Matching NLO QCD computations and parton shower simulations", JHEP 06 (2002) p. 29.

[15] S. Frixione, P. Nason and B.R. Webber, "Matching NLO QCD and parton showers in heavy flavor production", JHEP 08 (2003) p. 7.

[16] M. L. Mangano et al., "ALPGEN, a generator for hard multiparton processes in hadronic collisions", JHEP 0307 (2003), p. 1.

[17] S. Alioli, P. Nason, C. Oleari, and E. Re, "A general framework for implementing NLO calculations in shower Monte Carlo programs: the POWHEG BOX", JHEP 1006 (2010), p. 43.

[18] T. SjoİLstrand, S. Mrenna and P.Z. Skands, "A brief introduction to PYTHIA 8.1", Comput. Phys. Commun. 178 (2008) p. 852.

[19] G. Corcella et al., "HERWIG 6: An Event generator for hadron emission reactions with interfering gluons (including supersymmetric processes)", JHEP 0101 (2001), p. 10.

[20] M. Aliev, H. Lacker, U. Langenfeld, S. Moch, and P. Uwer: HATHOR, Comput. Phys. Commun. 182 (2011) p. 10341046.

[21] ATLAS Collaboration, ATLAS-CONF-2012-131, http://cds.cern.ch/record/1478370 .

[22] ATLAS Collaboration, ATLAS-CONF-2012-031, http://cds.cern.ch/record/1432196 .

[23] ATLAS Collaboration, ATLAS-CONF-2012-032, http://cds.cern.ch/record/1432198 . 
[24] ATLAS Collaboration, "Measurement of the top quark pair cross section with ATLAS in pp collisions at $\sqrt{s}=7 \mathrm{TeV}$ using final states with an electron or a muon and a hadronically decaying tau lepton", Physics Letters B717 (2012) p. 89.

[25] ATLAS Collaboration, "Measurements of top quark pair relative differential cross-sections with ATLAS in pp collisions at $\sqrt{s}=7$ TeV", Eur. Phys. J. C73 (2013) p. 2261.

[26] ATLAS Collaboration, "SUSY and Beyond SM Searches at ATLAS", these proceedings.

[27] https://twiki.cern.ch/twiki/bin/view/AtlasPublic/ExoticsPublicResults .

[28] https://twiki.cern.ch/twiki/bin/view/AtlasPublic/SupersymmetryPublicResults .

[29] ATLAS Collaboration, "Measurement of the t-channel single top-quark production cross section in pp collisions at $\sqrt{s}=7$ TeV with the ATLAS detector", Physics Letters B 717 (2012) p. 330.

[30] ATLAS Collaboration, ATLAS-CONF-2012-132, http://cds.cern.ch/record/1478371 .

[31] ATLAS Collaboration, ATLAS-CONF-2012-056, http://cds.cern.ch/record/1453783 .

[32] ATLAS Collaboration, "Evidence for the associated production of $a W$ boson and a top quark in ATLAS at $\sqrt{s}=7 \mathrm{TeV}$ ", Physics Letters B 716 (2012) p. 142.

[33] N. Kidonakis, "Next-to-next-to-leading-order collinear and soft gluon corrections for t-channel single top quark production", Phys. Rev. D 83 (2011) p. 091503.

[34] ATLAS Collaboration, "Search for tb resonances in proton-proton collisions at $\sqrt{s}=7$ TeV with the ATLAS detector", Phys. Rev. Lett. 109 (2012) p. 081801.

[35] ATLAS Collaboration, "Search for resonances decaying into top-quark pairs using fully hadronic decays in pp collisions with ATLAS at $\sqrt{s}=7$ TeV", JHEP 01 (2013) p. 116.

[36] ATLAS Collaboration, ATL-COM-PHYS-2008-008; https://cds.cern.ch/record/1082880?ln=en

[37] T. Plehn, G.P. Salam and M. Spannowsky, "Fat jets for a light Higgs", Phys. Rev. Lett. 104 (2010) p. 111801.

[38] T. Plehn, M. Spannowsky, M. Takeuchi and D. Zerwas, "Stop reconstruction with tagged tops", JHEP 10 (2010) p. 078.

[39] L.G. Almeida, S.J. Lee, G. Perez, G. Sterman and I. Sung, Template overlap method for massive jets", Phys. Rev. D 82 (2010) p. 054034.

[40] L.G. Almeida, O. Erdogan, J. Juknevich, S.J. Lee, G. Perez, et al., "Three-particle templates for a boosted Higgs boson", Phys. Rev. D 85 (2012) p. 114046.

[41] Y.L. Dokshitzer, G. Leder, S. Moretti and B. Webber, "Better jet clustering algorithms", JHEP 08 (1997) p. 001.

[42] M. Cacciari, G.P. Salam and G. Soyez, "The $\bar{k}_{t}$ jet clustering algorithm", JHEP 4 (2008) p. 63 [arXiv:0802.1189].

[43] ATLAS Collaboration, "A search for $t \bar{t}$ resonances in lepton+jets events with highly boosted top quarks collected in p-p collisions at $\sqrt{s}=7$ TeV with the ATLAS detector", JHEP 1209 (2012) p. 41.

[44] ATLAS Collaboration, "A search for $t \bar{t}$ resonances with the ATLAS detector in $2.05 \mathrm{fb}^{-1}$ of proton-proton collisions at $\sqrt{s}=7$ TeV", Eur. Phys. J. C 72 (2012) p. 2083.

[45] ATLAS Collaboration, "Measurement of the $W$ boson polarization in top quark decays with the ATLAS detector", JHEP 1206 (2012) p. 088.

[46] ATLAS Collaboration, ATLAS-CONF-2012-133, http://cds.cern.ch/record/1478373 .

[47] ATLAS Collaboration, ATLAS-CONF-2012-155, https://cds.cern.ch/record/1493494 . 